East African Medical Journal Vol. 77 No. 9 September 2000

NEONATAL INTESTINAL OBSTRUCTION IN ZARIA, NIGERIA

E. A. Ameh, MBBS, FWACS, Lecturer and Consultant and L. B. Chirdan, MBBS, Dip. Paed. Surg, FWACS, Lecturer and Consultant, Paediatric Surgery Unit, Department

of Surgery, Ahmadu Bello University Teaching Hospital, Zaria, Nigeria.

Request for reprints to: Dr. E. A. Ameh, Paediatric Surgery Unit, Department of Surgery, A.B.U. Teaching Hospital, Zaria, Nigeria.

\title{
NEONATAL INTESTINAL OBSTRUCTION IN ZARIA, NIGERIA
}

\author{
E. A. AMEH and L. B. CHIRDAN
}

\begin{abstract}
Objective: To study the aetiology, morbidity and mortality of neonatal intestinal obstruction. Design: A retrospective study.

Setting: Ahmadu Bello University Teaching Hospital, Zaria, Nigeria.

Subjects: One hundred and fifty one neonates ( $\leq 28$ days) undergoing surgery for mechanical intestinal obstruction.

Results: The male/female ratio was 3: 1 and median age at presentation was four days (range five hours - 28 days). Anorectal malformation was the commonest cause, 104 (68.9\%), 86.5\% of which were high anomalies and $13.5 \%$ low; the median age at presentation was three days. Fifty two per cent of colostomies for the high anomalies were performed using general anaesthesia and $48 \%$ local anaesthetic, but there was an increasing use of local anaesthesia over the years. Hirschsprung's disease accounted for 11(7.3\%) of the cases, representing $20 \%$ of all patients presenting with Hirschsprung's disease to this hospital; the median age was six days and in two patients the caecum and sigmoid colon respectively had perforated; nine patients had colostomy, one caecostomy and one ileostomy (total colonic aganglionosis). Eleven $\mathbf{( 7 . 3 \%}$ ) patients had incarcerated or strangulated ingunial hernia(ten) and congenital ventral hernia (one); the hernias were repaired in all patients and three required intestinal resection for gangrene, two of which had ipsilateral testicular gangrene, necessitating orchidectomy. Intestinal atresia was the fourth common cause of obstruction ten $(6.7 \%)$, eight of which were jejunoileal atresias and two duodenal and the median age was seven days; one atresia was associated with Hirschsprung's disease and had ileostomy, all other jejunoileal atresias were resected and duodenoduodenostomy was performed for the duodenal atresias. Other less common causes of neonatal intestinal obstruction were incarcerated exomphalos, malrotation, hypertrophic pyloric stenosis, annular pancreas, and idiopathic ileal volvulus and meconium ileus respectively. Postoperative complications occurred in sixteen of 95 patients $(\mathbf{1 6 . 8 \%})$ including colostomy or ileostomy complications 11 , wound infection three and anastomotic dehiscence(two). The overall mortality was $21.1 \%, 70 \%$ from overwhelming infection and $30 \%$ respiratory embarrassment; the mortality from the various conditions were Hirschsprung's disease $43 \%$, intestinal atresia $40 \%$, incarcerated exomphalos $40 \%$, anorectal malformation $18.5 \%$ and the only patient with volvulus died. Conclusion: The morbidity and mortality of neonatal intestinal obstruction in this hospital has improved over previous years due largely to meticulous resuscitation before surgery but the problems of late presentation and poor neonatal intensive care facilities persist. The findings are at variance with those in developed countries.
\end{abstract}

\section{INTRODUCTION}

Mechanical intestinal obstruction is one of the important conditions requiring emergency surgery in neonates. Momoh(1) had earlier reported on the pattern of neonatal intestinal obstruction in Zaria, Nigeria in which anorectal malformations, Hirschsprung's diseases and intestinal atresia respectively in that order were the common causes and mortality was high at $47 \%$. This is a retrospective report of the current trend of mechanical neonatal intestinal obstruction in Zaria, northern Nigeria.

\section{MATERIALS AND METHODS}

A total of 151 neonates (age $\leq 28$ days) were managed for mechanical intestinal obstruction at the Ahmadu Bello University Teaching Hospital, Zaria, Nigeria between January 1987 and December 1997 as identified from the operation registers. The operation notes, case notes and discharge summary sheets of the patients were analysed; all 151 patients were analysed for aetiology and treatment while further analysis of morbidity and mortality was limited to 95 with adequate records.

The evaluation of neonates with intestinal obstruction at the hospital is mainly clinical, supported by plain abdominal and 
chest radiography. Serum electrolytes and urea and haemogram are estimated routinely.

\section{RESULTS}

There were 113 boys and 38 girls with overall median age of four days (range five hours to 28 days) at presentation. The causes of mechanical intestinal obstruction in the 151 neonates are summarised in Table 1.

Table 1

Aetiology of neonatal intestinal obstruction in Nigeria

\begin{tabular}{lrrrr}
\hline \multicolumn{5}{c}{ Zaria } \\
Aetiology & This report & $\begin{array}{r}\text { Previous } \\
\text { report(1) } \\
(1982)\end{array}$ & *Ile-Ife(2) & Lagos(3) \\
& & $1992)$ & $(1976)$ \\
\hline Anorectal malformation & 68.9 & 63 & 49.4 & 16 \\
Hirschsprung's disease & 7.3 & 15 & 12.9 & 13.3 \\
Incarcerated/strangulated & & & & \\
hernia & 7.3 & 3.8 & 5.9 & 5.3 \\
Intestinal atresia & 6.7 & 7.5 & 22.4 & 24 \\
Incarcerated exomphalos & 3.3 & - & - & - \\
Malrotation (bands) & 2.6 & 3.8 & - & - \\
Hypertrophic & & & & \\
pyloric stenosis & 1.3 & 3.8 & - & 26.7 \\
Annular pancreas & 1.3 & - & - & 2.7 \\
Intestinal volvulus & 0.7 & 1.3 & 9.4 & 5.3 \\
Meconium ileus & 0.7 & - & - & - \\
\hline & & & & \\
\hline
\end{tabular}

*Hypertrophic pyloric stenosis not included

All figures are percentages

Anorectal malformation: This accounted for 104 (68.9\%) of the cases, 90 (69 boys, 21 girls) of which were of the high type and 14 (nine boys, five girls) low. The median age at presentation was three days (high three days, low two days) (range five hours to 28 days) the median weight was $2.8 \mathrm{~kg}$ (range $1.9-3.7 \mathrm{~kg}$ ). Patients with high malformations had colostomy; between 1987 and 1992, thirty one of the colostomies were performed under general anaesthesia and 17 using local anaesthetic, while from 1993 - 1997, $26(63.4 \%)$ were performed using local anaesthetia, nine (22\%) under general anaesthetia and in six (14.6\%) in which local anaesthesia was used initially, general anaesthesia was necessary due to technical difficulties. The treatment of low anomalies was by anoplasty(six), dilatation(six) and anal cut-back(two). In one boy, colostomy became necessary 48 hours after anoplasty due to increasing abdominal distension and failure to defaecate.

Five patients with high anomalies had other associated congenital anomalies as follows; oesoghageal atresia, ambiguous genitalia, cleft lip and palate and microcephalus, tibia vera and hypospadias each respectively.

Hirschsprung's disease: This occurred in eleven patients (7.3\%) and represents $20 \%(11 / 56)$ of all patients presenting to this hospital with Hirschsprung's disease during the period. All the patients were boys with a median age of six days (range four to twenty one days) at presentation. Eight patients had transverse colostomy and full-thickness biopsies taken from the rectum. In one of these patients, the ileum was injured during the procedure and was repaired. Two patients who had clinical evidence of peritonitis underwent laparotomy at which the caecum and sigmoid colon respectively were found to have perforated; the site of perforation was exteriorised as caecostomy in the patient with caecal perforation and sigmoid perforation was repaired and a defunctioning transverse colostomy constructed in the other, and biopsies were taken from the rectum. In all patients, aganglionosis was confirmed by histopathology.

Incarcerated and strangulated hernia: Eleven patients (7.3\%) had incarcerated or strangulated inguinal hernia (10 boys) and congenital ventral hernia (one girl). The median age was 23 days (range 15 - 28 days). The features at presentation were irritability, vomiting and tender, irreducible hernias. One patient had enterocutaneous fistula from necrosis. The duration of symptoms was six hours two days (median one day). One inguinal hernia was managed conservatively initially but this failed. Three patients (including one with ventral hernia) had bowel gangrene requiring segmental resection and anastomosis, both patients with inguinal hernias had ipsilateral testicular gangrene necessitating orchidectomy. The enterocutaneous fistula required resection. All hernias were repaired.

Intestinal atresia: Ten patients $(6.7 \%)$ had intestinal atresia at various levels; jejunoileal eight (five boys, three girls) and duodenum two (girls). The proximal dilated segment was gangrenous in patchy areas in three and perforated in one patient respectively with jejunoileal atresia. One patient with ileal atresia had associated total colonic aganglionosis while one with duodenal atresia had malrotation. The median age at presentation was seven days (range 5 -12 days) and median weight $2.8 \mathrm{~kg}$ (range $2 \mathrm{~kg}-3 \mathrm{~kg}$ ). Resection and anastomosis was performed for jejunoileal atresia and resection and ileostomy for one associated with total colonic aganglionosis. Duodenoduodenostomy was performed for the duodenal atresia along with a Ladd's procedure for associated malrotation in one.

Incarcerated exomphalos: In five neonates (3.3\%) aged 11 - 22 days (three boys, two girls), who were being managed non-operatively for major exomphalos, intestinal obstruction developed as a result of incarceration from adhesion between the bowel loops within the sac. There was no bowel gangrene in any patient. Treatment was by adhesiolysis and fascial closure of the defects.

Intestinal malrotation: Four patients (2.6\%) aged $5,12,14$ and 15 days respectively (two boys, two girls) presented with duodenal obstruction from bands as a result of intestinal malrotation. Ladd's procedure was performed in three patients and adhesiolysis and caecopexy in one. There was no recurrence in any patient.

Others: Other conditions causing intestinal obstruction in neonates included hypertrophic pyloric stenosis two 
(two boys aged 21 and 28 days), annular pancreas, two (a boy and a girl, aged five days respectively), idiopathic terminal ileal volvulus with bowel gangrene one (four-day old girl) and meconium ileus (20 hour old boy). Treatments were by pyloromyotomy, duodenoduodenostomy, intestinal resection and ileostomy respectively for the various causes. Records were not available regarding further investigation in the patient who had meconium ileus.

Morbidity and mortality: Postoperative complications occurred in 16 of 95 patients (16.8\%). There were complications in eleven of 61 colostomies (and ileostomy)(18\%) including separation five, retraction two, peritonitis two and stenosis two (nine had anorectal malformation and two Hirschsprung's disease); all the complications required re-operation including re-fashioning for separation, retraction and stenosis and laparotomy for peritonitis respectively. Three patients developed superficial wound infection following intestinal resection and herniotomy for strangulated inguinal hernias, which were controlled, by local wound care and antibiotics. Two other patients developed anastomotic dehiscence following resection and anastomosis for jejunoileal atresia; these required re-laparotomy, re-resection and anostomosis.

Table 2

Mortality in 95 neonates with intestinal obstruction

\begin{tabular}{lc}
\hline Condition & No. \\
\hline Anorectal malformation ( $\mathrm{n}=54)$ & 10 \\
Intestinal atresia ( $\mathrm{n}=10)$ & 4 \\
Hirschsprung's disease $(\mathrm{n}=7)$ & 3 \\
Incarcerated exomphalos $(\mathrm{n}=5)$ & 2 \\
Intestinal volvulus $(\mathrm{n}=1)$ & 1 \\
\hline
\end{tabular}

Figures in parenthesis are the number with adequate records

Twenty of ninety five patients $(21.1 \%$ ) died (Table 2), 14 from overwhelming infection (70\%) and six (30\%) respiratory impairment. The mortality rates for the various conditions were, Hirschsprung's disease $43 \%$ (caecal perforation, sigmoid perforation). intestinal atresia $40 \%$, incarcerated exomphalos $40 \%$, anorectal malformation $18.5 \%$ and the only neonate with ileal volvulus died.

\section{DISCUSSION}

The commonest cause of neonatal intestinal obstruction in this report is anorectal malformations (65\%) followed by Hirschsprung's disease as earlier reported(1) but incarcerated and strangulated inguinal hernias are now a more prominent cause. These findings are in contrast to those from Ile-Ife, western Nigeria(2) where anorectal malformation, intestinal atresia, Hirschsprung's disease and midgut volvulus respectively in that order are the common causes. However, in Lagos, western Nigeria(3) hypertrophic pyloric stenosis, intestinal atresia and anorectal malformation are the common causes (Table 1). In a report from north America(4), Hirschsprung's was the second most common cause of neonatal intestinal obstruction after necrotising enterocolitis. While in developed countries the diagnosis of Hirschsprung's disease is being increasingly made in the neonatal period(5), the reverse is the situation in Zaria, where the proportion of these patients presenting in the neonatal period has fallen from $56 \%$ (1) to $20 \%$ in the present report. In developed countries, intestinal atresia is a common cause of congenital intestinal obstruction(6-8) and accounted for one-third of causes of neonatal intestinal obstructions in one report(8). In Zaria, however, only $6.7 \%$ of cases of neonatal intestinal obstruction are due to intestinal atresias. Reports from developed countries have also shown midgut volvulus from malrotation to be a frequent cause of intestinal obstruction in the newborn $(9,10)$ and in Myanmar(11), a developing country, $24.4 \%$ of children with volvulus were neonates. In Zaria, neonates account for $18 \%$ of children with volvulus(12). These differences may well be due to the fact that many neonates with intestinal atresia and volvulus in our environment do not reach hospital.

The median age at presentation in this report was four days, and patients with anorectal malformations presented particularly late. This is similar to other reports from Nigeria(1,2). Most of our patients had frank features of intestinal obstruction and required only plain abdominal radiographs (and cross-table lateral radiographs or invertogram) for diagnosis. Barium enema was helpful in patients with Hirschsprung's disease without peritoneal signs. Plain supine and erect abdominal radiographs are frequently the only investigations necessary for the confirmation of intestinal obstruction in neonates(13), contrast studies being reserved for diagnostic difficulties, and then in the absence of acute peritoneal findings.

The overall morbidity in this report was $17 \%$, mostly (69\%) from colostomy. The mortality of $21 \%$ compares to $47 \%$, in a previous report(1) from the same centre and $44.6 \%$ and $33 \%$ respectively $(2,3)$ from other parts of Nigeria. The reduction in mortality in this report may be due to meticulous resuscitation and more use of local anaesthetic for colostomy in patients with anorectal malformation. The problems of lack of facilities for neonatal intensive care and total parenteral nutrition however remain. This is in contrast to developed countries where mortality from the common causes of neonatal intestinal obstruction has reduced $(5,8)$, mostly due to earlier presentation and availability of improved neonatal support facilities. It is hoped that the outcome in our environment will improve further as facilities improve and more patients present early.

\section{ACKNOWLEDGEMENTS}

To Professor P.T. Nmadu for allowing us to include his patients in this report, the many residents who contributed to the management of the patients and the medical records department of the hospital for providing the records. 


\section{REFERENCES}

1. Momoh J.T. Pattern of neonatal intestinal obstruction in Zaria, northern Nigeria. East Afr. Med. J. 1982; 59:819-823.

2. Adejuyibge O., Jeje E.A., Owa J. and Adeoba E.A. Neonatal intestinal obstruction in lle-lfe, Nigeria. Nig. Med. J. 1992; 22:24 -28 .

3. Olumide F., Adedeji A. and Adesola A.O. Intestinal obstruction in Nigerian children. J. Paediat. Surg. 1976; 11:195-204.

4. Klein M.D., Coran A.G., Wesley J.R. and Drongowski R.A. Hirschsprung's disease in the newborn. J. Paediat. Surg. 1984; 19:370 - 374.

5. Philoppart A.1. Hirschsprung's disease. In: Ashcraft KW, Holder TM (eds). Paediatric Surgery, WB Saunders Company, Philadelphia, 1993:305 - 319.

6. Dillon P.W. and Cllleg R.E. Newborn Surgical emergencies: Gastro intestinal anomalies and abdominal wall defects. Paediat. Clin. N. Amer. 1993; 40:1289 -139.
7. Lister J. Development in neonatal surgery 1995 -1980. J. roy Coll. Surg. Edinb. 1980; 25:324 - 332.

8. Touloukian R.J. Intestinal atresia and stenosis. In: Ashcraft K.W., Holder T.M. (eds), Paediatric Surgery, WB Saunders Company, Philadelphia, 1993: 305 - 319.

9. Powel D.M., Othersen H.B. and Smith C.D. Malrotation of the intestines in children: effect of age on presentation and therapy. $J$ Paediat. Surg. 1989; 24:777 - 780.

10. Welch G.H., Azmy A.F. and Ziervogel M.A. The surgery of malrotation and midgut volvulus: a nine-year experience in neonates. Ann. roy. Coll. Surg. Engl. 1983; 65:244 - 246.

11. Maung M. and Saing H. Intestinal volvulus: an experience in a developing country. J. Paediat. Surg 1995; 30:679-681.

12. Ameh E.A. and Nmadu P.T. Intestinal volvulus: aetiology, morbidity and mortality in Nigerian children,Paediat. Surg Int. 2000;165:5052 .

13. Atwell J.D. Neonatal intestinal obstruction. In: Atwell J.D. (ed), Paediatric Surgery, Anorld, London, 1998:197-204.

Letter to the Editor-in-Chief

Dear Sir

\section{RE: PLEOMORPHIC ADENOMA OF THE HUMAN BREAST}

Pleomorphic adenoma is the commonest tumour of the major salivary glands(1). A similar tumour has been described in the skin and breast (2-4). The tumour may be misdiagnosed radiographically, cytologically and by frozen section as a malignant lesion(4). A review of the English literature only revealed 54 reported cases.

Recently we encountered two pleomorphic adenomas of the breast. The patients aged 41 and 54 years respectively. They both presented with painless, hard and highly mobile masses without axillary lymph node enlargement. Both patients were treated with excisional biopsies.

Grossly the two specimens measured $3 \mathrm{~cm}$ and $5 \mathrm{~cm}$ respectively. The masses were well circumscribed and surrounded by dense connective tissue. (Figure 1). On cutting both masses had a gritty sensation; resulting from extensive calcification. Cut surfaces revealed a white gray to tan appearance. Microscopic examination revealed benign looking tumours surrounded by fibrous pseudocapsules. Three components of the tumour were identified. There were sheets and cords of cells with plasmacytoid features and containing abundant eosinophilic cytoplasm.

\section{Figure 1}

Cut surface of pleomorphic adenoma showing a glistering surface

In areas the tumour cells were arranged in glandular structures lined by cuboidal epithelial cells. The surrounding stroma was myxoid with extensive chondroid metaplasia. Neither cellular pleomorphism nor mitoses figures could be demonstrated in any of the tumours.
The epithelial cells were positive with cytokeratin and epithelial membrane antigen. The myoepithelial cells were positive for Vimentin, S100 protein and muscle specific actin. One patient was lost to follow up and the other is tumour free after 13 months follow up. The differential diagnosis of this rare tumour include phylloides tumour with chondroid metaplasia. The latter tumour will, however, not contain any myoepithelial cells. Owing to the presence of calcifications, a pleomorphic adenoma can easily be confused with a carcinoma radiologically. The latter tumour should show cellular anaplasia and mitotic figures which are lacking in pleomorphic adenoma.

Another tumour to be considered in the differential diagnosis is myoepithelioma. This tumour is composed of pure proliferation of myoepithelial cells without chondroid or osseous metaplasia.

The histogenesis of pleomorphic adenoma of the breast remains controversial. Several observers have proposed that these tumours arise from the ductal epithelium. The subareolar location of these tumours and associations with intraductal papillomas in some of the cases seem to strengthen this school of thought. Having seen a similar association, Smith and Taylor contended that this tumour was a papilloma with mesenchymal metaplasia(5).

Other authors advocate that pleomorphic adenomas arise from stem cells with a divergent differentiation. These stem cells differentiate into epithelial, myoepithelial and mesenchymal elements. The author is in agreement with this theory of histogenesis.

Pleomorphic adenoma of the breast is a benign tumour. Only three cases with recurrences have been reported and there is no report of malignant differentiation in these lesions. The treatment of these lesions is surgical excision with a thin rim of normal tissue. A simple enucleation is not recommended as it may lead to recurrence.

Finally, both clinicians and pathologists should be aware of the diagnosis of pleomorphic adenoma to avoid unnecessary radical surgery. As recurrences have been reported a close follow up of the patients is recommended.

Yours sincerely

M. N. Muthuphei, MBChB, MMed, FFPath, MPhil, Senior Specialist/Senior Lecturer, Department of Anatomical Pathology, Medical University of Southern Africa, P.O. Box 93909, Boordfontein 0201, South Africa.

\section{REFERENCES}

Rengezi, J.A. and Scuibba, J.J. Oral pathology: Clinical-pathologic correlations 2nd edition. WB Saunders. 1993; 265-267.

McKee, P.H. Pathology of the skin with clinical correlations. 2nd edition. MosbyWolfe. 1996; 15.64-15.66.

Chen, K.T.K. Pleomorphic adenoma of the breast. Amer. J. Clin Pathol.

4. McKek, M. and Von Hochstetten, A.R. Pleomorphic adenoma of the human breast. J. Surg. Oncol. 1980; 14:281-286.

Smith B.H. and Taylor H.B. The occurrence of bone and cartilage in mammary tumours. Amer. J. Clin. Pathol. 1969; 51:610-618. 